\title{
Tangence
}

\section{Enquête sur l'identité : l'instance policière dans Copies conformes, La mauvaise foi et Paye-moi une bouffe, poète!}

\section{Pierre L'Hérault}

Numéro 38, décembre 1992

Fiction policière et roman actuel

URI : https://id.erudit.org/iderudit/025734ar

DOI : https://doi.org/10.7202/025734ar

Aller au sommaire du numéro

Éditeur(s)

Tangence

ISSN

1189-4563 (imprimé)

1710-0305 (numérique)

Découvrir la revue

Citer cet article

L'Hérault, P. (1992). Enquête sur l'identité : l'instance policière dans Copies conformes , La mauvaise foi et Paye-moi une bouffe, poète !. Tangence, (38), 8-23.

https://doi.org/10.7202/025734ar d'utilisation que vous pouvez consulter en ligne. 


\section{Enquête sur l'identité : l'instance policière dans Copies conformes, La mauvaise foi et Paye-moi une bouffe, poète!}

Pierre L'Hérault

Javais lu pour le plaisir, au moment de leur parution, c'est-à-dire presque d'affilée, Copies conformes de Monique LaRue, La mauvaise foi de Gérald Tougas et Paye-moi une bouffe, poète! de MarieFrançoise Taggart ${ }^{1}$. Une deuxième lecture, pour les besoins d'un cours mais non dénuée pour autant de plaisir, transforma en conviction l'impression de la première que la présence de l'instance policière dans les trois romans était plus qu'une coïncidence. Ne s'agissait-il pas plutôt d'une conjonction significative à laquelle ne serait pas étranger le caractère emblématique reconnu, par la critique et les distinctions, à ces récits marquant le passage entre les décennies quatre-vingt et quatre-vingt-dix? Le métissage formel, résultat du croisement des romanesques canonique et policier ne signalait-il pas, en effet, au delà des singularités de chaque texte, leur appartenance à une esthétique postmoderne, seule convenable à des récits moins axés sur la recherche de l'identité que sur sa problématisation, qui seraient moins une quête de l'identité qu'une enquête sur elle? J'ai cru trouver un appui à mon hypothèse chez Uri Eisenzweig, qui me servira de guide principal dans cette lecture provisoire. Je retiendrai surtout de son Récit impassible que, comme "texte illusoire", le récit policier conduit à *parler de sa périphérie, du discours social qui le commente, qui le pose, suppose, propose ${ }^{2}$ et qu'il est

[...] dans la logique textuelle d'une certaine littérature "postmoderne - d'avoir recours à l'univers du hard-boiled, où la question du pouvoir narratif est posée mais (à la différence du récit policier classique) non résolue; où l'identité est dépourvue de

1 Monique LaRue, Copies conformes, roman, Montréal, Lacombe, 1989; Marie-Françoise Taggart, Paje-moi une bouffe, poète!, roman, Montréal, VLB éditeur, 1990; Gérald Tougas, La mauvaise foi, roman, Montréal, Québec/Amérique, coll. *Littérature d'Amérique ", 1990. Dans mon texte, je renverrai à ces ouvrages par une simple mention de la page entre parenthèses.

2 Uri Eisenzweig, Le récit impossible. Forme et sens du roman policier, Paris, Christian Bourgois, 1986, p. 12. 
psychologie, mais non de cohérence (autre contraste avec le modèle générique); où la réflexion n'est plus en représentation, mais en acte; où la Vérité, donc, loin d'être avant que d'être perçue, s'identifie à la perception elle-même. ${ }^{3}$

Par la façon dont ils intègrent les éléments du policier, nos trois textes correspondent à la situation décrite par Eisenzweig. Qu'ils ne se présentent ni ne soient reçus *uniquement comme récits d'énigme ou de suspense, mais comme littérature à part entière " ${ }^{4}$, la chose est claire. D'une part, tout ou presque de leur paratexte s'emploie, sinon à dénier, du moins à minimiser l'appartenance à un * mauvais " genre. D'autre part, ils sont publiés par des maisons d'édition qui ne se spécialisent pas dans le paralittéraire; au Devoir comme à La presse, ils sont pris en charge par les grands titulaires de la chronique littéraire: Jean Royer et Réginald Martel 5; Tougas reçoit le prix du Gouverneur général, et LaRue le Grand Prix du Livre de Montréal; Taggart voit consacré par Martel son "sacré tempérament d'écrivain ${ }^{6}$. Afin de préciser les rapports que le littéraire entretient dans ces textes avec le policier, je m'intéresserai d'abord au paratexte et à la réception critique, ensuite j'examinerai la réutilisation du policier dans chaque roman; et, finalement, reprenant une expression d'Eisenzweig, je chercherai le "sérieux massif sous l'ironie discrète "?

\section{Paratexte et réception}

Une seule quatrième de couverture, celle de Copies conformes, parle de "l'intrigue policière", mais à la toute fin et

$3 \quad$ Ibid., p. 293.

4 Selon les termes du libellé de l'atelier où fut donnée une première version de ce texte: «L'esprit du genre ou le roman policier et sa réutilisation dans le texte littéraire contemporain. (Association des professeurs de français des universités et collèges canadiens (APFUCC), Sociétés savantes, Queen's University, Kingston, 28 mai 1991). Voir Nouvelles de l'Association des professeurs de français des universités et collèges canadiens, vol. IX, $\mathrm{n}^{\circ} 1$ (automne 1990), p. 4. Cet article se rattache également, par sa problématique, au groupe de recherche . L'identitaire et l'hẻtérogène dans la prose romanesque québécoise depuis 1940*, dont je suis membre. Ce groupe est subventionné par le CRSH du Canada.

5 J'y reviendrai plus loin.

6 Réginald Martel, - Taggart en version originale couleurs et stéréo, La presse, 18 novembre 1990 , p. C-5.

7 Op. cit., p. $42:$ : [...] à l'image de ce qui se passe dans bien des récits de détection, la vérité se cache à la surface des choses, et sous l'ironie soidisant discrète on retrouve un sếrieux tout à fait massif. . 
comme un élément parmi d'autres. Si le premier paragraphe y reconnaît l'intertexte de Dashiell Hammett, c'est pour le mettre au service d'autre chose: l'interrogation d'une jeune femme sur "l'apparente banalité de sa vie *; la "recherche de disquettes perdues ou volées" entraînerait dans un "monde étrange, qui est peut-être le nôtre *. En somme, à travers un "dédale ensoleillé ", l'on "mène l'intrigue policière avec jubilation *, mais on "parle avec force de l'amour, de la maternité, de la langue et du sentiment d'appartenance 8 . Des propos graves, peu compatibles en apparence avec le polar, qui le débordent en tout cas; mais la référence est ici à Hammett. Ce qui oblige à nuancer considérablement les choses 9 . Dans la publicité subséquente à l'obtention du Grand Prix du Livre de Montréal 1990, sur lequel donc on misera, il ne sera nullement fait état de la parenté avec le roman policier. On retiendra une citation de Marie-Claude Fortin qui insiste sur "l'intelligence qui émane de l'écriture" ${ }^{10}$; une

8 Il faudrait mentionner cependant l'illustration de la couverture qui flirte avec le genre policier et populaire. Elle se présente comme une sorte de collage aux formes géométiques irrégulières jouant sur les contrastes d'un rose devenant saumon, puis presque orange, d'un vert tendre devenant vert feuille et d'un gris (beaucoup moins important en volume) passant au noir. À l'avant-plan et en gros plan, légèrement déportée vers la gauche, une grosse décapotable américaine; à l'arrièreplan et presque estompée, coupant à mi-hauteur de l'illustration à la manière d'une bande blanche pigmentée de noir et collée, le profil d'une ville moderne sur fond rose; entre la voiture et la ville, deux hauts palmiers en vert feuille se découpant sur le même fond rose. Dans le coin inférieur droit, un losange gris et noir, au centre duquel se trouve un cercle blanc délimité par un fort trait noir et auquel s'ajoute un court trait noir de même intensité. Ces deux derniers éléments pourraient suggérer l'ouverture du canon d'un revolver vu par la personne sur laquelle il serait braqué. Dans le coin inférieur opposé, à l'intérieur d'une forme géométrique irrégulière et horizontale de couleur orange se trouve intégré à la composition le mot * Roman .

9 Car, considéré comme le créateur du bard-boiled novel, il *a introduit un fond social dans les histoires policières. (Stefano Benvenuti, Gianni Rizzoni et Michel Lebrun, Le roman criminel. Histoire, auteurs, personnages, trad. de l'italien par Cécile Supiot, Nantes, L'Atalante, 1982, p. 104). Raymond Chandler a ainsi défini sa manière: « Hammett a remis l'assassinat entre les mains des gens qui le commettent pour des raisons solides et non pour foumir un cadavre à l'auteur. [...] Il colla ces gens sur le papier tels qu'ils sont dans la vie et il leur donna le style et les réactions qui sont habituellement les leurs, dans des circonstances donnécs. = (Ibid., p. 100) C'est son plus célèbre roman, The Maltese Falcon (Le faucon maltais), 1930, qu'utilise LaRue.

10 Marie-Claude Fortin, "Le vol du faucon ", Voir, 23-29 novembre 1989, p. 24. 
deuxième, de Jean Royer qui parle d'un "roman fascinant des années 1980. Résolument moderne et féminin, intelligent et raffiné. Décapant *11; et une troisième, de Guy Cloutier, où il est question de "quelque chose de profondément mûri dans l'émotion comme dans l'écriture que seuls quelques grands d'ici ont atteint .12.

Pour les deux autres textes, rien sur la quatrième de couverture qui fasse allusion au roman policier. Dans le cas de $\mathbf{L a}$ mauvaise fot, on nous en détourne même soigneusement, diraiton, en parlant d'un auteur qui *se transforme en biographe passionné " de l'xhistoire de la vie et de la mort d'Irène [quil est aussi l'histoire de celui qui, longtemps après, la raconten. L'élucidation d'une mort, comme moteur de l'action, cède le pas au « retour au pays natal ", c'est-à-dire à l'aventure initiatique du narrateur. La réception critique emboite le pas. Elle décourage quand elle n'interdit pas de chercher ailleurs que dans cette direction. Royer écrit - ce avec quoi d'ailleurs je ne puis qu'être d'accord:

Ce livre de Gérald Tougas porte le genre romanesque à un sommet de finesse et d'élégance, de culture et de plaisir littéraire. En même temps qu'il propose la peinture sociale d'une époque - celle des années 50 au Manitoba et des années 60 au Québec - ce roman nous montre un écrivain à la recherche de lui-même. 13

Et il s'étend sur la "passion de la littérature" de Tougas et sur ses lectures: Flaubert, Valéry, Hugo, Shelley, Coleridge, Byron, Chateaubriand, Anne Hébert, Grandbois, Saint-Simon, Proust, SaintDenys Garneau, Miron... ${ }^{14}$ Martel mentionne le roman policier, mais pour indiquer que celui de Tougas échappe à ce genre, devenant *vite une vaste enquête, non pas policière au sens strict mais qui en emprunte et en adopte un peu les méthodes, pour savoir, et comprendre si possible, ce qui est arrivé à la sœeur du narrateur"15. Il s'agit bien "d'un chef-d'œuvre, à placer sur le même rayon que les quelques grands livres de la littérature

11 Jean Royer, *Adieu, femmes romanesques, Copies conformes", Le devoir, 18 novembre 1989 , p. D-8.

12 Guy Cloutier, Avec Copies conformes, Monique LaRue joint son nom aux plus grands écrivains d'ici •, Le soleil, 25 novembre 1989, p. F-11.

13 Jean Royer, Gérald Tougas. La mauvaise foi de toute foi ", Le devoir, 2 février 1991, p. D-4.

14 Ibid.

15 Réginald Martel, "La mauvaise foi, une œuvre majeure, La presse, 21 octobre 1990, p. C-2. 
québécoise et étrangère " ${ }^{16}$. Pour sa part, même si elle parle "d'une cuvre complexe de reconstitution, mosaïque fragile dont le sujet ne se révélera dans son entier qu'à la toute fin ", Fortin n'emploie pas l'expression "policier", parlant d'un roman qui "accomplit admirablement son travail de roman "17. Lucie Côté non plus, du reste, qui retient surtout l'aspect autobiographique du roman et "la mémoire et [...] la culture prodigieuses " de l'auteur ${ }^{18}$.

Qu'en est-il de Paye-moi une bouffe, poète! de Taggart? La quatrième de couverture joue sur la "grâce "19, le "ton" et le -talent - étonnants de cette auteure qui n'a que vingt-deux ans. À la jeunesse, pourquoi ne pas associer la "couleur", les "rebondissements ", l' "humour "? Qu'en disent les critiques? Martel y reconnaît le "roman western américain ", pointe, à côté de la verve et du rythme, "une certaine gravité du propos" 20 qui lui donnerait des lettres de noblesse. Le critique J. Gagnon, dont le jugement négatif est l'exception, n'y voit qu'«une assiette renversée, toutes choses éparses ", qu'une "foule de situations et de personnages non résolus „21. Encouragée par l'auteure qu'elle interviewe, Lucie Côtét ${ }^{22}$ établit une filiation entre l'écriture de Taggart et les grands auteurs que lui lisait son père lorsqu'elle était enfant: Hugo, Balzac, Melville, Daudet, Pagnol... Mais a-t-elle suffisamment noté que cette liste force les cadres de la tradition française et de la grande littérature par l'introduction de Melville et de Pagnol? A-t-elle surtout accordé assez d'importance au fait qu'à la question de savoir ce qu'est un écrivain, son père répond par une définition

\section{Ibid.}

17 Marie-Claude Fortin, "La grande noire sœur", Voir, 29 novembre5 décembre 1990 , p. 24.

18 Lucie Côté, .Gérald Tougas: le temps était venu d'écrire *, La presse, 28 octobre 1990 , p. C-5.

19 Jouée à fond par la photo couleur pleine page de la première de couverture: une jeune femme blonde (l'auteure), nonchalemment assise sur un banc public, les bras croisés sur le dossier du banc et sur lesquels s'appuie la tête, se tournant donc vers quelqu'un qu'elle semble attendre (un lecteur, une lectrice?) en le fixant du regard, et tournant le dos à une terrasse vide a laquelle fait face le banc.

20 Réginald Martel, *Taggart en version originale couleurs et stéréo , La presse, 18 novembre 1990 , p. C-5.

21 J. Gagnon, .Paye-moi une bouffe... Plat du jour *, Voir, 7-13 mars 1991, p. 23.

22 Lucie Côté, *Depuis l'enfance, l'écriture s'est frayé un chemin " La presse, 10 mars 1991, p. C-5. Toutes les citations, textuelles ou non, données dans ce paragraphe sont tirées de cet article. 
qui retient davantage le critère "consommation "que le critère "valeur littéraire": "[...] c'est celui qui écrit les livres qu'on lit"? C'est pourtant à cette définition que l'auteure attribue sa résolution d'écrire: "[...] je veux faire ça plus tard! . Si elle ne parle pas du roman policier, Taggart ne fait nullement problème ni mystère de sa fréquentation des mauvais genres, comme de la grande littérature. Se voisinent ici Calvino, Marie-Claire Blais, la bande dessinée western: "Je voulais justement que ça fasse BD.

La rapidité de cette enquête force à reconnaître que l'appartenance ou même l'apparentement avec le genre policier est à la fois reconnu et nié par les diverses instances de production, de réception, de circulation et de légitimation: aucun des trois romans ne se présente comme un roman policier; un seul, Copies conformes, sur la quatrième de couverture, annonce explicitement l'élément d'intrigue policière; l'un, La mauvaise foi, détourne l'enquête policière au profit de la *biographie"; les critiques les reçoivent et les jaugent comme des textes littéraires, de même que les instances de légitimation.

\section{L'utilisation du policier}

Le roman policier est pourtant un intertexte réel et significatif de ces trois romans: il importe donc de chercher à préciser l'usage qui en est fait par chacun. Reconnaissons d'abord que la position des auteur-e-s est beaucoup plus nuancée que celle qu'on a pu dégager du discours du paratexte et de la critique. S'ils ne font pas du policier proprement dit, mais se réclament de la grande littérature, on ne trouve pas chez eux cette sorte de prévention, qui semble parfois être celle de certains critiques contre le genre. Ils s'en servent au contraire avec un savoir-faire qui montre qu'ils en sont des lecteurs et lectrices assidus, y trouvent non seulement l'évasion, mais des matériaux, des structures et des motifs qu'ils n'hésitent pas à utiliser au même titre que s'ils les prenaient aux classiques, non comme une forme dégradée d'intertextualité.

Monique LaRue en use de façon intensive et explicite, en instrument maîtrisé de l'écriture. L'intertextualité policière est d'abord signalée par l'épigraphe de chacune des trois parties, citations tirées du Faucon maltais de Dashiell Hammett, sur 
14

lequel elle construit son roman. S'ensuit un rapport minutieux de correspondances qui, en un sens, fait de Copies conformes une lecture-relecture-réécriture du texte de l'inventeur américain du roman noir, jusqu'à en mimer certains éléments. C'est le cas de la scène finale du suspense, qui entraînera le personnage principal dans l'hôtel et la suite même où, dans Le faucon maltais, s'est produit le meurtre. Claire Dubé entre dans le roman de Hammett; elle y entre très consciemment, puisqu'elle l'a lu. Phénomène de lecture dédoublé plusieurs fois, dans lequel la lectrice de Hammett, personnage du roman que je lis, se voit devenir personnage du roman de Hammett qui m'est ainsi donné à (re)lire. Enregistrons l'utilisation hyperconsciente du policier par LaRue qui a comme effet, à travers la convention générique, de souligner l'écriture en fabrication. Par la distanciation, le policier est conduit à autre chose qu'à lui-même. L'intrigue policière, en fait, double l'intrigue de Copies conformes en lui servant de miroir. La donnée essentielle du roman de LaRue est la suivante: à une semaine du retour prévu à Montréal, Claire Dubé, 35 ans, reste seule à San Francisco, avec son fils Philippe, 5 ans, son mari ayant dû rentrer précipitamment à cause de la maladie fatale de sa mère. Ces situations de rupture (couple, enfant, mort) avivent l'interrogation identitaire (de la femme, du couple, de la Québécoise) poursuivie au cours de ce séjour d'un an à l'étranger. Sur cette situation se greffe le récit de détection, lui-même partiellement modelé sur l'intrigue du Faucon maltais: retrouver une disquette, volée ou perdue, qui contient les recherches de pointe de son mari. Ainsi, à cause de l'intervention de l'intrigue du Faucon maltais, le roman donne lieu à une série de poursuites, de doubles, de dissimulations, devient l'enquête sur soi favorisée par le déplacement en lieu étranger.

Aucune trace explicite d'intertextualité policière dans $L a$ mauvaise foi. L'intertextualité affichée est celle des grands textes (mais de différentes traditions). Comme LaRue, cependant, Tougas accorde beaucoup d'importance à l'acte de lecture. Dès le début du roman, il inverse les perspectives. L'enquêteur n'est plus un personnage du roman, celui sur qui repose l'intrigue qu'il conduit, mais le lecteur. Lisons ce passage:

Dans la voiture du passé, si je te racontais l'histoire de ma sœur Irène, sa mort, ce que j'en sais, ce qu'on m'en a dit, peut-être comprendrais-tu pourquoi en te la racontant, je te l'ai déjà dit, j'ai l'impression de remonter à l'origine de mon grand dérangement, tu te débrouilleras, tu es payé pour ça, spécialiste de la lecture à 
l'envers, peut-être me diras-tu pourquoi, spécialiste de l'amour et de la mort, la belle tête de Christine me fait irrésistiblement penser à celle de ma sœur Irène dans son cercueil [...l, et tant qu'à y être tu me diras, si tu peux, comment elle est morte, si c'était un accident, un meurtre ou un suicide. (p. 24-25)

Le tu est ambigu: il commence par désigner la jeune fille anglophone avec qui le narrateur voyage et finit par se confondre avec le lecteur/la lectrice, qui, à la faveur de cette ambiguité, s'entend demander deux choses: expliquer le personnage narrateur à luimême, à partir des bribes de sa biographie, c'est-à-dire jouer le rôle du psychanalyste; et trouver le "comment " de la mort d'Irène, c'est-à-dire jouer le rôle de l'enquêteur policier. En d'autres mots, les procédés du roman policier serviront à écrire le récit du "voyage initiatique dans le passé" (p. 24). À propos de la mise en évidence, par le recours au policier, du rôle de lecture que joue l'écriture, il faudrait encore considérer la place considérable que prend le récit de l'oncle Philippe qui apparaît, au lecteur comme aux personnages de la famille qui l'écoutent, comme une promesse de résolution de l'énigme. Comme l'enquêteur, Philippe ne vient-il pas d'ailleurs et n'est-il pas le plus fin et logique causeur? Mais justement, son récit bute sur l'énigme et le comment de la mort. Un beau discours, certes, mais de mauvaise foi, pour autant qu'il promet plus qu'il ne donne, qu'il se clôt sur lui-même, masquant, par cette compensation verbale, son impuissance. La promesse non tenue du roman policier servirait-elle ici d'appât au lecteur/à la lectrice? Se servirait-on en fait d'un langage connu et aimé pour lui parler d'autre chose, tout en renouvelant sans en avoir l'air le très vieux roman de la quête? Dans cette perspective, on pourrait être tenté d'inverser la très juste formule de Renald Bérubé et dire que le roman de Tougas n'est pas seulement le «lieu mobile d'une enquête", mais aussi le "moment d'une quête" 23 . Gardons-nous-en et gardons à l'endroit la formule de Bérubé: Tougas subvertit avec beaucoup d'habileté le roman de la quête, sans remplacer tout uniment la quête par l'enquête. Dans ce détournement plein de subtilités et de clins d'œil aux grandes traditions littéraires occidentales réside une part de l'intérêt et de la réussite de son texte.

23 Renald Bérubé, *Éclats et grâces de La mauvaise foi *, Voix et images, vol. XVI, $\mathrm{n}^{\circ} 3$ (printemps 1991), p. 548: "votre voyage en train, cher Marcel, n'est pas seulement le moment d'une quête, il est aussi le lieu mobile d'une enquête $[. .$.$] ».$ 
Chez Marie-Françoise Taggart, le policier est l'une des formes de roman et d'écriture moderne populaire qui, avec la bande dessinée et le western, voisinent avec Calvino, Cortazar, MarieClaire Blais. Grande lectrice de romans quétaines de série noire ou rose, son personnage Catherine vit avec un É crivain (je souligne le grand $\bar{E}$ ) et partage l'amitié, entre autres, d'un bédéiste. Le roman concilie les fréquentations antinomiques du personnage et de la narratrice, Voyons dans cette coexistence "matérielle " de formes hétéroclites une rupture intentionnelle et objective avec une vision, aussi bien moderne que classique, de la littérature. L'Écrivain ne s'appelle-t-il pas Babiche? Ce qui pour moi évoque la babiche dont on tissait le fond des chaises canadiennes, donc le folklore, le patrimoine. Et du patrimoine, je glisse au patriarcat dont Babiche, dans son acte d'écrire, est l'indéniable et naïf représentant, ainsi qu'on peut le voir dans la "Séance d'écriture" du chapitre III. Dans un monde urbain d'images de cinéma tendres et violentes défilant au gré de la marche, de flashes, d'éclatement, de mouvement, il est difficile, comme chez Tougas, de dire les choses en empruntant le schéma initiatique, ou, comme chez LaRue de percevoir le .fil du temps. (p. 9). Il y a chez eux une distance spatiale et temporelle - donc une possibilité de recul - qu'on n'a pas ici où l'on ne dispose que de l'instant. L'épisode policier, dont sort indemne Catherine, permet au récit western de prendre sa place et d'assurer la suite. Le mot *FIN * est le "signe que tout ce qui est terminé recommence inlassablement . (p. 176). Les dernières lignes du livre sont:
[...] ouverture plus que fermeture tout en gardant le cachet mythologique du cow-boy (dans ce cas-ci il s'agirait plutôt d'une cow-girl) qui s'éloigne sur les chemins brûlés de l'Ouest, chevauchant le bon vieux cheval, parfois même jouant un air sur sa guitare, en sifflant sur une fausse note mais arrivant tout de même à faire pleurer tous les spectateurs, à les faire espérer une prochaine aventure, à les faire imaginer ce qui se passera ensuite... (Ibid.)

Le roman se termine donc sur des points de suspension. On retrouverait ici la série, caractéristique du policier (et du paralittéraire en général) qui, en pays états-unien croise, se confond même infailliblement, dans la vie comme sur les écrans, avec le western. Par sa finale, où elle fait intervenir la série, Taggart réaffirme très explicitement le rapport de son texte au genre populaire. Rapport pas du tout naif, puisque le western-policier lui sert à construire son texte, ainsi donné à lire comme un travail de lecture et de 
réécriture. Il lui permet en outre de soutenir un propos grave que contient l'intrigue ramenée à sa plus simple expression: Catherine réussit à s'échapper des griffes de son pimp et cherche à se desserrer celles de son patriarcal amant Babiche. L'élément policier, que ce soit le suspense ou l'enquête, renvoie constamment à l'écriture ou à la lecture d'un livre. Babiche lui-même, dans tous ses récits, même quand il les croit hautement fabulateurs, est infailliblement ramené à l'autobiographie: à se demander s'il n'a pas tué sa sœur. Au moment où Catherine est poursuivie par son mac, elle lit, comme par hasard, le passage d'un roman où Betty est elle-même poursuivie. On pourrait croire que le livre la protège. L'écriture serait une poursuite permettant d'échapper à une autre poursuite. N'est-ce pas finalement le schéma policier-western qui structure le texte de Taggart?

\section{Le sérieux massif sous l'ironie}

Mais quel est donc, pour reprendre l'expression d'Eisenzweig, le "sérieux tout à fait massif " sous "l'ironie soi-disant discrète du policier ${ }^{24}$ ? Il s'agit d'un double propos qui se conjugue: l'un sur l'écriture et l'autre sur l'identité et l'origine.

Le premier me suggère les considérations suivantes. L'hybridité et l'ironie des formes mises en relief par le policier apparaissent comme une marque de la postmodernité des textes lus, postmodernité non réductible cependant à ce seul facteur. Il s'agit d'un indice, celui d'une intertextualité qui n'est plus seulement celle des grands auteurs, mais aussi celle des genres dits "mauvais ", "mineurs", "populaires", etc. Cela ne caractérise-t-il pas justement l'attitude postmoderne, telle qu'on la comprend aujourd'hui? Cela est vrai des trois romans, mais selon des modes différents. Les trois œuvres étudiées permettent de saisir trois types d'intertextualité qui eux-mêmes renvoient à trois âges de l'histoire québécoise ${ }^{25}$. Parlons donc d'âge, sans manquer à la discrétion toutefois, puisque nous nous en tiendrons à ce qui est du

24 Voir note 7.

25 Je ramène ici très librement à la dimension de la microhistoire littéraire québécoise les propos de Janet Paterson s'appliquant à l'histoire littéraire générale, dans Moments postmodernes dans le roman québécois (Ottawa, Les Presses de l'Université d'Ottawa, 1990). J'ai utilisé surtout le chapitre 5: *Intertextualités dans Le semestre.. 
domaine public ${ }^{26}$. Au moment où il publie La mauvaise foi, Tougas, à cinquante-sept ans, a suivi un parcours assez peu linéaire. Originaire du Manitoba, formé dans un collège classique québécois, soumis aux perturbations, enthousiasmes, engagements et libérations de la Révolution tranquille, il combine la référence à la grande tradition de lecture gréco-latine, française, mais aussi anglaise (à laquelle l'introduit un professeur d'anglais passionné) et même négro-africaine (grâce à un séjour de quatre ans au Niger) aux théories et stratégies textuelles les plus actuelles. LaRue, à l'instar de son personnage Vasseur, viendrait-elle d'une famille «en transition" (p. 26)? Elle avait douze ans en 1960, vingt en 1968. Formée aux "humanités", puis à la philosophie, puis à l'analyse du discours, dans la mouvance de la Révolution tranquille et de la contestation, elle vient à l'écriture à la fin des années soixante-dix, davantage donc à l'âge du texte, de l'intertexte (de l'intertexte québécois, puisqu'il est devenu possible) et de la traduction qu'à celui de la parole; à l'âge de l'éclatement et du fractionnement des certitudes collectives, sous l'effet entre autres du discours féministe. Le livre et la lecture occupent toujours dans son roman, bien que sans exclusive (le langage de l'ordinateur y joue), la place centrale. Marie-Françoise Taggart, à vingt-deux ans, est la seule des trois à être née après l'avènement de la télévision au Québec. Il n'est donc pas étonnant que son texte fasse une place aussi grande à l'image animée et à la bande dessinée western, brisant sans équivoque l'hégémonie de la référence au livre. Je n'irai pas plus loin dans ce petit jeu par lequel j'ai voulu seulement suggérer que l'écriture a aussi ses âges (social et biologique). Il me plaît bien de constater que le moment de publication de ces trois romans est celui de la coïncidence, de la coexistence, du croisement et du cumul de trois âges. La rencontre de la diachronie et de la synchronie crée un moment postmoderne particulièrement significatif en ce qu'il sert de révélateur à l'hétérogénéité de l'espace textuel québécois. Il y a en effet quelque chose d'exemplaire dans le fait que ces trois textes, si différents, soient écrits, lus et reçus dans un même espace-temps, sans qu'il y ait gommage de l'un par l'autre; dans le fait que des pratiques

26 En plus des quatrièmes de couverture, les informations sont tirées des sources suivantes. M. LaRue: Réginald Hamel, John Hare et Paul Wyczynski, Dictionnaire des auteurs de langue française en Amérique du Nord (Montréal, Fides, 1989); M.-F. Taggart: l'article déjà cité de L. Côté; G. Tougas : les articles déjà cités de L. Côté, R. Martel et J. Royer. 
d'écritures aussi autonomes se côtoient sans s'annuler: de la pratique intégrative et hiérarchisante de Tougas, qui soumet l'esthétique postmoderne à l'épreuve de l'esthétique et de la mémoire classiques, où le livre occupe toute la place, au jeu très maîtrisé de l'hybridité formelle de LaRue, où le livre occupe encore le centre, mais comme fabrication, jusqu'aux fréquentations formelles douteuses de Taggart, dans lesquelles le livre perd son exclusivisme intertextuel. Les trois se rejoignent cependant dans cette autre caractéristique fondamentale de la postmodernité: l'ìmpossibilité du récit, que justement viendrait mettre en valeur, selon Eisenzweig, le roman policier. J'entre par là dans le deuxième propos annoncé touchant à l'identité et à l'origine.

Au terme de son aventure, la narratrice-personnage de Copies conformes formulera ainsi le questionnement identitaire: «Et ces voix de mes amies, et les romans que je lis l'hiver, avaient fini par construire en moi une femme que je n'étais pas, et que le soleil de la Californie m'avait permis d'apercevoir. ( p. 189) L'instance policière, en permettant le jeu de lecture déconstructive, permettrait ici à l'identité d'être montrée à la fois comme ce qui est à trouver et comme ce qui se dissimule, fuit, éclate ${ }^{27}$. Non pas comme un tout donné, mais comme la poursuite d'un objet existant mais inatteignable, dont on reconnaît les traces, mais qui s'échappe. Il y a fuite dans les deux sens du mot. L'objet fuit devant la poursuivante; il fuit aussi comme une "fuite d'eau ". En cours de route, il se modifie. Les travaux du mari de Claire ne portent-ils pas sur l'interlangue, c'est-à-dire la *langue de transition * (p. 13)? D'où la question soulignée par l'intrigue policière: comment distinguer l'original de la copie?

Dans La mauvaise foi, la recherche de la résolution de l'énigme (la mort de la sœur du narrateur) joue sur la construction spatio-temporelle du roman, elle-même élaborée sous l'impulsion de la force d'attraction identitaire et héréditaire. Elle permet de retrouver des lieux, des moments, mais toujours dans leur dimension de fuite, d'ailleurs, dans leur ambiguité de lieux et

27 Cette vision tensionnelle de l'identité est très aiguë dans Copies conformes. Suggérons-le en opposant à la phrase précitée les suivantes: "[...] je n'ai jamais pensé qu'on puisse sortir de sa langue natale si facilement. J'étais la femme d'une seule langue. (p. 34); "Je n'avais jamais partagé l'illusion qu'on puisse choisir sa vie. Mais je désirais être fidèle à ce qui avait eu lieu. Je n'aime pas les gens qui forcent le destin. (p. 65) 
de temps imaginés. Le narrateur est conscient: «Je souris en pensant que c'est assis dans le sens inverse de la marche que je vais poursuivre mon voyage initiatique. (p. 24) Tourné, non pas vers où l'on va (d'où l'on vient, en fait), mais vers où l'on vient (d'où l'on est). Ici se trouve réalisê ce qu'Eisenzweig attribue de constitutif au roman policier: "Par conséquent, la lecture ellemême ne peut qu'effacer le début du récit, sa différence fondatrice $[\ldots]_{*}^{28}$. L'effort de lire le récit de la mort d'Irène ne rend-il pas le passé du narrateur et du lecteur inatteignable? À travers la mort d'Irène, ne dit-on pas le départ sans retour: la mort à sa communauté, peut-être même la mort de sa communauté d'origine elle-même? Se confondant temporellement avec le départ de Marcel, la mort d'Irène interdit le retour au "pays natal ". Rappelons ici le propos d'Eisenzweig cité en introduction: "Car qu'estce que parler d'un texte illusoire [...] sinon, avant tout, parler de sa périphérie, du discours social qui le commente, qui le pose, suppose, propose?" 29 . L'enquête fait surgir le discours social qui émerge, sous l'éclairage des propos de l'oncle Philippe, à travers le récit de la fameuse scène où le conseil de famille présidé par le curé demande à Irène de rompre avec Eric, à travers aussi l'évocation des rapports sociaux et des préjugés d'un milieu rural franco-manitobain des années cinquante, etc. Ironiquement, c'est même par la génétique que l'identité éclate: "Renoir vit avec une métisse de la Coulée. Elle est superbe, plus belle que Francmiche. Ils ont un petit garçon d'une douzaine d'années, un petit garçon qui a une tête d'Inuk ou de Vietnamien ébloui. . (p. 266)

Pour le personnage de Taggart, le rapport à l'identité se présente d'une façon différente, mais toujours en relation avec un point d'origine, celui du père. Betty, la jeune fille de Colorado Springs, dont Catherine lit l'aventure, a été chassée du *bercail paternel * (p. 62) à cause d'une fugue. L'écrivain Frédérick Babiche, qui revient toujours à l'autobiographie, vient d'ailleurs, a un passé mystérieux et parle "de ce père qui se décomposait $[. .$.$] avant$ même d'achever sa vie * (p. 13). L'importance du lieu d'origine, du lieu qu'on a quitté, je la trouve corroborée par l'interview de Taggart à Lucie Côté ${ }^{30}$. Me gardant loin de la psychocritique, je

28 Le récit impossible, p. 11.

29 Ibid., p. 12.

30 Lucie Côté, *Depuis l'enfance, l'écriture s'est frayê un chemin*. Toutes les citations qui suivent sont tirées de cet article. 
noterai simplement que l'auteure y précise que *Taggart est un nom écossais*, que son "père est un Américain d'une petite ville du Colorado ", cette petite ville, ajoute-t-elle, *dont je parle dans mon livre ". C'est la deuxième fois dans l'interview qu'il est question du père. Il en sera question une troisième fois, quand l'auteure raconte sa grande * déception d'enfance * qui a été d'apprendre que son père était *Anglais *, selon l'expression utilisée. Le père avait si bien masqué son origine que la petite fille l'ignorait. Est-ce pour cela qu'il faut utiliser l'enquête policière, pour démasquer le "faussaire", le "traitre "? En d'autres mots, pour retrouver une part de son identité, ce trésor perdu, la bourse ramassée par erreur par Betty (l'auteure apprend par accident que son père est "Anglais") et qui va motiver la poursuite de la part du propriétaire. Il y a appropriation de l'imaginaire paternel du Colorado. Ce roman ne se lit donc pas sans reconnaître la centralité du rapport au père. Ce père qui, en la lui lisant, lui transmet la grande littérature; qui lui donne une définition déterminante de l'écrivain; qui a quitté son Colorado natal pour "aller étudier le dessin animé et la bande dessinée en Europe ; qui s'est finalement établi à Montréal, où *il a vraiment choisi de vivre en français". Comment démêler cet écheveau identitaire, encore compliqué du fait que l'on écrit dans la langue de la mère? Voilà l'énigme qui suffit à l'« enquête policière ", qui imprime au roman son hybridité et fait de Montréal un espace "carrefour" (p. 173), celui de la violence urbaine rappelée par les gros titres d'Allo police, mais aussi celui où l'on peut pleurer en entendant la "romance" (p. 174) du cow-boy, de la cow-girl plutôt, et où l'on peut vivre avec un vrai écrivain. En somme, il s'agit ne pas défaire l'écheveau identitaire, d'en jouer...

\section{Conclusion}

Dans ces trois romans, soulignant l'éclatement du récit, son incertitude, sa nécessaire pluralité, son impossible cloisonnement, l'instance policière apparaît comme la marque d'une esthétique qui cherche, dans les concordances et les discordances des voix, à faire entendre le réel fractionné et multiple. La structure policière serait donc reprise davantage pour sa fonction interrogative que pour sa capacité à résoudre une énigme. Cette façon postmoderne de construire le roman permet de problématiser l'identité, de la voir, selon l'expression de LaRue, comme une "construction" hétérogène. Je cite Copies conformes: «Nous ne sommes pas des 
acteurs" (p. 190). Mais il y a acteur et acteur! Sans vouloir contredire LaRue, je citerai Josée Dupuy qui soutient que, dans la série noire, *on quitte le point de vue de l'enquêteur pour prendre celui d'un acteur du drame à venir "31. Ainsi l'hybridité formelle, vue ici à partir de l'utilisation du roman policier, rejoint-elle et confirme-t-elle ce que nous avons défini ailleurs ${ }^{32}$ comme une caractéristique de l'écriture des années quatre-vingt: l'irruption de l'hétérogène dans la fiction. Ces romans se rattachent, dans leur fabrication même, à une conception des choses où la culture est vue, non comme une complétude donnée, mais comme un espace dynamique où les permutations et combinaisons d'éléments donnent lieu à des jeux de construction qui ne sont pas plus gratuits que l'enquête policière qui, cherchant à remonter, en toute logique, à la cause et aux mobiles, ne trouve en fait que le présent et sa complexité (dont fait partie le rapport à l'origine).

Le recours au policier manifeste ici une autre tendance du roman québécois actuel: sa revendication de l'espace américain. La référence de LaRue à Hammett prend alors toute sa portée. On reconnaît en effet à ce dernier d'avoir donné une forme américaine au roman policier ${ }^{33}$. Or, c'est justement dans ce contexte

31 Josée Dupuy, Le roman policier, Paris, Librairie Larousse, 1974, p. 43.

32 Dans Sherry Simon, Pierre L'Hérault, Robert Schwartzwald et Alexis Nouss, Fictions de l'identitaire au Québec (Montréal, XYZ éditeur, 1991), ouvrage collectif du groupe de recherche :L'identitaire et l'hétérogène dans la prose romanesque québécoise depuis 1940 .

33 " "Hammett a rompu totalement avec l'influence des auteurs anglais", a reconnu Ellery Queen lui-même, "et il nous a donné le premier un roman policier américain à cent pour cent. Il n'a pas inventé un nouveau type d'histoires policières, mais il a inventé une façon nouvelle de les raconter" * (Benvenuti et al., op. cit., p. 102). C'est dans les termes suivants que LaRue situe son roman par rapport à celui de Hammett: - L'intrigue a quelque chose du roman policier et je le vois comme un hommage à Hammett qui est un grand écrivain américain, cynique et désabusé. (Jean Basile, * Amour, micro-ordinateur et suspense à San Francisco., La presse, 2 décembre 1989, p. K-1). Par ailleurs, elle pose ainsi la question de l'américanité: - Il se trouve tout simplement que mon héroïne, montréalaise et francophone naturellement, est confrontée avec un monde très différent de ce qu'elle a connu. Elle est placée, par les circonstances, face à des images auxquelles elle ne peut pas s'identifier, qu'elle ne peut même pas comprendre. C'est d'autant plus curieux que nous sommes américains aussi. Le décalage entre les Américains américains et nous semble très léger, mais il est plus profond qu'il n'y paraît. $\mathrm{Au}$ fond, comment rester soi-même quand on est francophone américain?" (ibid) 
d'un déplacement à travers l'Amérique que LaRue l'utilise. Il est aussi un certain langage américain dont se nourrit le texte québécois. Plus encore: ce langage américain (dans le sens étatsunien) est reconnu comme fabricateur de l'identité québécoise. Montréal et San Francisco, ce sont deux espaces, deux langages étrangers, mais deux espaces que relient une interlangue, justement l'objet des recherches du mari de Claire Dubé. Cela est très immédiatement perceptible dans Paye-moi une bouffe, poète! où est mis à contribution le langage western, ce "naturel américain du Québécois qui revient au galop* ${ }^{34}$, selon l'expression de Jean Larose. Qu'il soit tout à coup nommé, retrouvé dans son origine coloradienne me semble une autre marque de cet imaginaire transfrontalier et métissé qui est en train, non pas de naître - il existe depuis longtemps —, mais d'être admis et utilisé consciemment comme matériau. Ainsi en est-il de l'espace canadien de Tougas qui, fût-il impossible, n'en est pas moins, et peutêtre à cause de cela, pour le personnage un espace constructeur en ce qu'il continue de s'imposer comme une énigme, l'énigme qui empêche la fermeture, la clôture. La mawvaise foi n'existe pas sans la lecture du poète américain Robert Frost sur laquelle se noue l'intrigue amoureuse et tragique qui motive l'enquête policière. Chacun de ces romans constitue une traversée de l'Amérique ou, plus justement, se laisse traverser par le langage et l'espace américains, selon le rapport dialogique variable exprimé par le chiasme bakhtinien * mien-étranger/étranger-mien . 35. Mais écrire au Québec, n'est-ce pas nécessairement cela? Au delà de la thématique, des références explicites ou implicites à l'espace et aux auteurs américains, le recours au genre policier constitue la signature américaine des romans de LaRue, Taggart et Tougas.

34 Jean Larose, L'amour du pauvre, Montréal, Boréal, 1991, p. 92. Tiré du texte * Le cheval du réel - [1989] qui porte sur la efigure exceptionnelle * (p.89) de Will James proposée par Jacques Godbout dans son film Alias Will James (ONF, 1988).

35 Mikhaïl Bakhtine, les carnets. 1970-1971 *, Esthétique de la création verbale, Paris, Gallimard, 1979, p. 365. 